\title{
Immunotherapy
}

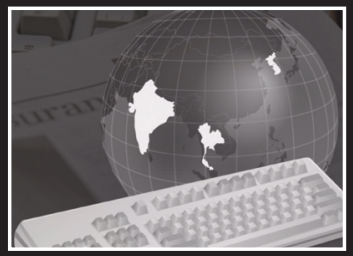

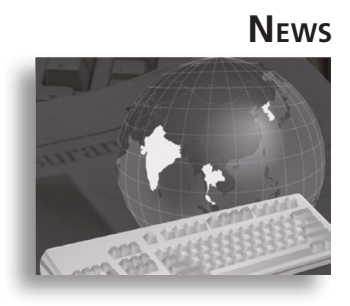

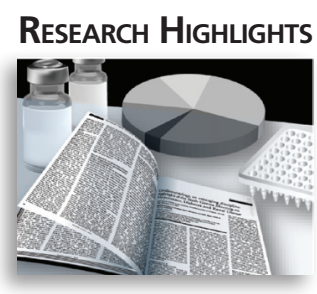

In recent research published in Nature Immunology, Amnon Altman (La Jolla Institute for Allergy and Immunology, CA, USA) and fellow investigators discovered a molecular interaction that is necessary for the activation of $\mathrm{T}$ lymphocytes. It is thought that this finding could lead to a better understanding of autoimmune diseases and allergies.

About the discovery, Mitchell Kronenberg (La Jolla Institute for Allergy and Immunology) explains: "Dr Altman's finding is a breakthrough in our understanding of the complex biochemical changes that trigger the immune system's T lymphocytes, which are disease-fighting white blood cells, to mount an immunological attack." By blocking this newly discovered cellular interaction, it may be possible to develop new treatments for autoimmune diseases such as rheumatoid arthritis and multiple sclerosis.

Previous research has demonstrated that when $\mathrm{T}$ cells see a foreign antigen, an enzyme known as protein kinase $\mathrm{C}-\theta$ (PKC- $\theta)$ is recruited to the immunological synapse. The molecular mechanism of the PKC- $\theta$ recruitment and how necessary this process is for T-cell activation to occur correctly was still unknown.

In the present study, when researchers set out to map the section of PKC- $\theta$ enzyme that is involved in its 'colocalization' with the immunological synapse, they found an essential physical interaction in this process occurred between PKC- $\theta$ and T-cell stimulatory receptor, CD28. Regarding this previously unknown physical interaction, Altman states: "We identified a small region of the enzyme, called the V3 region, that binds CD28, and demonstrated that this binding is critical for the enzyme's unique localization and its function."

According to Altman, drug companies have been trying to create small-molecule drugs that block the activity of the PKC- $\theta$ enzyme, as the importance of the enzyme in T-cell activation was already known. However, Altman states: "Here, we have found an alternative way of blocking the function of PKC- $\theta$, not by inhibiting its enzymatic activity, but by inhibiting its recruitment to the immunological synapse, which is that part of the cell where it needs to be to activate $T$ cells. Essentially, the enzyme remains fully active, but it can't trigger T-cell activation because it's not in the right place in the cell."

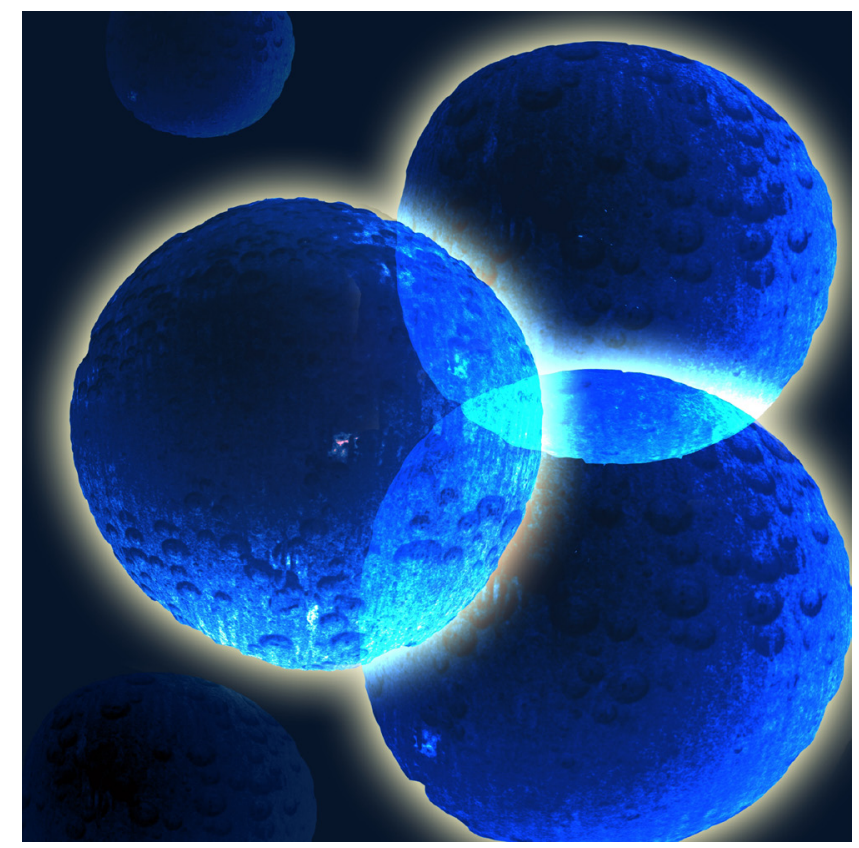




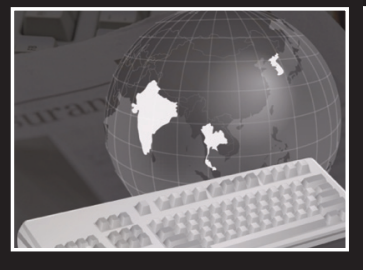

Referring to the study by Altman and colleagues, Christopher Rudd (University of Cambridge, UK) states: “Dr Altman has mapped a novel interaction between PKC- $\theta$ and CD28, and has shown that this specific region modulates the immune system response to antigens. This now means that this region in PKC- $\theta$ can be targeted by novel therapeutics, or that protein fragments of the region itself could be used to treat a variety of immune disorders." Indeed, authors of the study wrote that their results "indicate V3-based 'decoys' may be therapeutic modalities for T-cell-medicated inflammatory diseases."

It is probable that the process for targeting the interaction between CD28 and PKC- $\theta$ will be highly selective and thus undesirable side effects on other cells and tissues will be few, as the PKC- $\theta$ enzyme and CD28 are only expressed together in T cells.

Altman explains that it is not easy to design a method to block the PKC- $\theta$ enzyme binding to CD28: "Several possibilities exist, such as using a peptide to block the interaction. However, getting a peptide into a $\mathrm{T}$ cell is not a trivial matter. While we consider our finding very promising, we know that developing a therapeutic to block this interaction is not something that will happen next year. It will take time, but the potential is exciting."

\section{- By Roshaine Gunawardana}

Sources: Kong KF, Yokosuka T, CanonigoBalancio AJ, Isakov N, Saito T, Altman A. A motif in the V3 domain of the kinase PKC- $\theta$ determines its localization in the immunological synapse and functions in $T$ cells via association with CD28. Nat. Immunol. 12(11), 1105-1112 (2011); La Jolla Institute for Allergy \& Immunology Press Releases: www.liai.org/pages/news-releases_oct_2_2011b

\section{AIMSPRO ${ }^{\circledR}$ shows promise for treatment of scleroderma}

Recently announced results of a Phase II trial have suggested Daval International's AIMSPRO $^{\circledR}$ to be safe and well tolerated as a monotherapy to patients with late-stage established diffuse cutaneous systemic sclerosis.

AIMSPRO is a new-generation peptide/ polyclonal biological product, derived from hyperimmune caprine serum raised against an inactivated HIV IIIB viral lysate. The double-blind, placebo-controlled study evaluated the effects of AIMSPRO $4.5 \mathrm{mg} / \mathrm{ml}$ twice weekly compared with placebo. The primary aim of the study was to evaluate safety and tolerability of AIMSPRO in the treatment of 20 patients with systemic sclerosis for a period of 26 weeks of study participation.

At the end of this period, there were no signs of deterioration in hematological, cardiological, biochemical or immunological parameters measured. In addition to encouraging safety signs, AIMSPRO also showed signs of clinical benefit in secondary outcome measures including the Modified Rodnan Skin (MRS) Score,
Scleroderma UK Functional Score, Patient and Physician Global Assessment (VAS), the Short Form 36 (SF-36) and the MRC Sum Score. The MRS score provides a validated measure of disease severity, and in the patients receiving placebo, a worsening was recorded (changes: $-27.07 \%$; $\mathrm{p}=0.29$ ) whereas a stability was observed in AIMSPRO-treated patients. Furthermore, patients receiving AIMSPRO demonstrated a distinct improvement in the overall SF-36 scores (changes: $+41.6 \% ; \mathrm{p}=0.184$ ).

The results also demonstrated trends towards benefit for lung function measures. The forced expiratory volume in $1 \mathrm{~s}\left(\mathrm{FEV}_{1}\right)$ decreased in the placebo group compared with baseline at 26 weeks (change: $-5.6 \%$; $\mathrm{p}=0.0582$ ). By contrast, no deterioration was observed in the AIMSPRO-treated group. Similarly, at 26 weeks, the placebo group showed a decrease in the forced vital capacity (FVC) compared with baseline (change: $-5.6 \%$, $\mathrm{p}=0.1038)$, whereas there was an increase in FVC in the AIMSPRO-treated group (change: $+1.8 \% ; \mathrm{p}=0.3225$ ).
"Although requiring further confirmatory studies, these results are quite exciting, especially when compared with what has been seen in other pilot trials in scleroderma as there is a clear unmet medical need for patients suffering with this life-threatening disease," enthused Christopher Denton, Principal Investigator of the trial and Professor of Experimental Rheumatology at the Royal Free Hospital in London, UK. "The important value of the safety data from such a well-conducted trial in such a serious disease is clear, apart from the signals of therapeutic benefit."

Daval International is now assessing the biomarker data collected in this clinical trial and correlating this with the changes observed, so as to shed more light into the mechanism of action of AIMSPRO in the context of late-stage established diffuse cutaneous systemic sclerosis.

\section{- By Paolo Reveglia}

Sources: Daval International News \& Resources: www.davalinternational.com/news/news_5. html; ClinicalTrials.gov: http://clinicaltrials.gov/ ct2/show/NCT00769028

\section{About the News and Views}

The News and Views highlights some of the most important events and research. If you have newsworthy information, please contact: Duc Hong Le, Editor, Immunotherapy Future Medicine Ltd, Unitec House, 2 Albert Place, London, N3 1QB, UK; Tel.: +44 (0)20 83716090 ; Fax: +44 (0)208343 2313; d.le@futuremedicine.com 


\section{Innate lymphoid cells: new role in repairing influenza-induced tissue damage}

Innate lymphoid cells (ILCs) are a heterogeneous group of cells that has an important role in immunity and inflammation in the intestine, but their roles at other mucosal sites remain poorly understood. In a recent report, David Artis and his team from the Perelman School of Medicine at the University of Philadelphia (PA, USA) have showed that mice lacking ILCs suffered from "loss of airway epithelial integrity, diminished lung function and impaired airway remodeling" following influenza infection compared with normal mice.

\footnotetext{
"Innate lymphoid cells (ILCS) are a heterogeneous group of cells that has an important role in immunity and inflammation in the intestine, but their roles at other mucosal sites remain poorly understood."

Further analyses of these ILCs from the experimental mouse lungs showed that they expressed immune-related proteins such as Thy-1 (CD90), IL-2 receptor $\alpha$-chain (CD25), IL-7 receptor $\alpha$-chain (CD127) and IL-33 receptor subunit T1-ST2. Importantly, these ILCs also produced a growth factor called amphiregulin. Administration of amphiregulin or infusion of ILCs to the lungs of infected mice lacking ILCs could normalize lung
}

function, suggesting that the activation of these cells is critical to tissue repair at lung surfaces.

Of note, the research team also found that ILCs do not attack the virus per se as other immune cells do; rather, they activate the proliferation of cells that line the lung, which aids in wound healing of the lung tissues that have been severely damaged as a result of the viral infection. As such, the authors proposed that the ILCs' effect on lung wound healing and tissue remodeling may also be seen in other respiratory infections and possibly in noninfectious lung injury and inflammation such as asthma.

"The identification of innate lymphoid cells in the lung, and new studies from multiple research groups illuminating their previously unrecognized functions in diverse disease processes could help in the design of new drugs to prevent or better fight many common infectious or inflammatory diseases," commented Artis.

- By Emily Tulk

Sources: Monticelli LA, Sonnenberg GF, Abt $M C$ et al. Innate lymphoid cells promote lung-tissue homeostasis after infection with influenza virus. Nat. Immunol. doi:10.1031/ ni.2131 (2011); University of Pennsylvania News Release: http://health.upenn.edu/news/ News_Releases/2011/10/immune-cells 